\title{
Principles of integrated agricultural systems
}

Rapid changes occurring in the agricultural environment are placing increased demands on producers. To respond to these demands, farmers need to manage their systems by reducing risk, while retaining management flexibility. Integrated agricultural systems have the potential to meet these objectives. Integrated agricultural systems are whole-farm strategies and technologies organized to help producers manage multiple enterprises in a synergistic manner to improve profitability and natural resource stewardship. In the past, American agriculture was focused solely on its ability to produce sufficient food, fuel and fiber to meet national and global demands. Agriculture has been largely successful in meeting these production demands. While productivity will continue to be a major factor in food production systems, increased societal demands for environmentally sound management, the need for rural community viability and a rapidly changing global marketplace are now shaping the evolution of more integrated and sustainable agricultural systems. In their efforts to help design and manage these systems, researchers need to be aware of the impact resulting from these external influences.

This special issue on 'Principles of Integrated Agricultural Systems' presents a hierarchical structure of agricultural systems, sets forth working definitions of these systems and describes their external influences. The goal for this special issue was to construct a framework to develop agricultural management systems that rely more on renewable resources, such as solar capture, biologically mediated nutrient cycling and pest control and crop-animal interactions, and less on non-renewable resources, such as fossil fuels and application of synthetic fertilizers and pesticides.

Principles of integrated agricultural systems need to be developed for several reasons. First, principles can be used to organize thinking. Understanding how a system is structured and how it functions is imperative when a system is investigated. Development of simulation models and decision support systems cannot be accomplished without first analyzing the fundamental processes controlling the system and defining how the processes are interrelated. Second, principles can be used to develop new management systems. Once the underlying principles of a system are understood, various functional components can be restructured to form new systems. Third, principles can be used to develop hypotheses to drive research. For example in the paper by Archer et al., panelists identified research needs that could be expanded into researchable hypotheses, including (1) defining the relationship between agricultural policy and human health, (2) identifying the connection between obesity and the cost of healthy foods and (3) ascertaining whether the continual pressure on farms to become larger might be directly linked to vertical integration within the commercial sector. A set of guiding principles for integrated agricultural systems was initiated at a workshop held in Mandan, North Dakota, in 2004. This workshop in the northern Great Plains was the first in a series of workshops. Subsequent meetings were held in Alabama in 2005 and in Maine in 2008.

In this special issue, four general categories were used to describe the influences on agricultural systems: (1) social/ political, (2) economic, (3) environmental and (4) technological. These papers suggest that the following critical issues must be addressed by agricultural managers, policy makers and researchers. First, sustainable systems must be flexible enough to respond to the future challenges facing agriculture. Second, newly emerging social and political factors include rising fuel costs, obesity, potential decreases in commodity subsidies, consumer awareness and demands to know how food is produced, and economic returns to land. Third, American agriculture operates in a market-driven economy that is impacted by policy, technology and environmental concerns. These factors in turn affect the scale of operation, while controlling management flexibility. Fourth, agricultural incentives control management decisions, often to the detriment of the environment. Integrated agricultural systems need to be developed that are adapted to the environment, rather than trying to adapt environmental constraints to the agricultural systems. Fifth, enhanced technology has increased the complexity of farming. Sixth, future agricultural systems must address emerging issues in land use, decline in work force and societal support of farming, global competition, changing social values in both taste and convenience of food, and increasing concerns for food safety and the environment. Seventh, a set of robust principles needs to be defined and subsequently used to design adaptable integrated agricultural systems. 
During the past several decades, several changes have occurred in the structure and operation of farm and ranch systems, thereby impacting the continued sustainability of American agriculture. These changes include a reduction in the number of farms and farmers, increases in farm size, and concomitantly the commercialization of farms. New policies must be developed to encourage the integration of agricultural systems. Farmers want to produce the most marketable and cost-effective products. Therefore, they logically choose to produce what they perceive as the 'best' commodities resulting in increasing specialization without much regard for the environment. Actively managing for multiple services can substantially reduce agriculture's environmental footprint and can be encouraged with production incentives that reward environmental stewardship. These incentives, whether trade-based or policy-based, must be tailored to forestall continued environmental degradation and loss of agricultural sustainability. Policies with adequate incentives must be provided for ecosystem services, such as clean water and air, productive and healthy soil, habitat development and restoration, and carbon sequestration and storage.

Current American agricultural systems are dramatically different from agricultural systems at the start of the 20th century. Economic, social/political, environmental and technological drivers have all interacted to shape the current agricultural domain. To understand the structure of future agricultural systems, an understanding of how drivers have affected current agricultural systems is needed. Highly specialized systems, such as commodity crop production and supply chain livestock production, appear most vulnerable to future changes. Future agricultural systems need to be developed to balance multiple goals and ensure sustainability. We believe that a dynamic set of integrated agriculture production principles and practices will allow producers the flexibility to achieve this balance. This special issue should help to stimulate further discussion on the topic and put forth a path towards developing and achieving agricultural sustainability.

Jon D. Hanson USDA-ARS, Northern Great Plains Research Laboratory, 1701, 10th Avenue, SW

PO Box 459, Mandan,

ND 58554, USA jonhanson@ars.usda.gov

Alan Franzluebbers USDA-ARS, J. Phil Campbell Sr. Natural Resource Conservation Center 1420, Experiment Station Road, Watkinsville, GA 30677, USA 